\title{
Efficacy of Direct Injection of Ethanol into the Myocardium to Control Aconitine-induced Ventricular Tachycardia in Anesthetized Dogs
}

\author{
Akira Kurita, MD, FACG, Hideki Mrtani, MD, \\ Ritushi Kato, MD, Hiroyuki Hikita, MD, \\ Toshihiko Nishioka, MD, Bonpei TAKaSe, MD, \\ Hirokazu Nagayoshi, MD, Akimi Uehata, MD, \\ and Kimio SatOMura, MD
}

\begin{abstract}
SUMMARY
The effect of injecting ethanol directly into the myocardium to control aconitine-induced ventricular tachycardia (VT) was evaluated in anesthetized dogs. In 17 dogs, VT was induced by injecting aconitine $(1.0 \mu \mathrm{g}$, median dose) directly into the epicardium. After inducing persistent VT for up to $3 \mathrm{~min}, 0.6$ $\mathrm{m} l$ (median volume) of $96 \%$ ethanol was injected into the same epicardial region. Regular sinus rhythm reappeared in 15 dogs with no change in systolic blood pressure; the other 2 dogs died of ventricular fibrillation (VF). In another $13 \mathrm{dogs}$, VT was induced by injecting aconitine directly into the endocardium using a Variocath needle catheter. After persistent VT for up to $3 \mathrm{~min}$, a regular sinus rhythm was restored in 7 dogs by injecting $2.0 \mathrm{ml}$ (median volume) of $96 \%$ ethanol; the remaining 6 dogs died of VF. Histology showed no transmural necrosis and the subendocardial necrotic areas were essentially the same in the dogs that recovered from VT as in those that died. There was no statistically significant relationship between doses of ethanol and VT duration. These preliminary results suggest that the injection of ethanol into the myocardium may efficiently terminate VT when other techniques fail. (Jpn Heart J 1996; 37: 611-625)
\end{abstract}

Key words: Chemical ablation Ventricular tachycardia Ethyl alcohol

$\mathrm{P}$ ATIENTS with sustained ventricular tachycardia (VT) are at high risk of sudden cardiac death when the arrhythmia cannot be controlled by antiarrhythmic drug therapy and/or devices. ${ }^{1,2)}$ Treatment of VT by radio-frequency catheter ablation is less promising than the ablation of accessory and/or dual atrioventricular (AV) pathways. ${ }^{3-6)}$ Further, surgical intervention to inflict a lesion by direct myocardial resection, or to stimulate the myocardium by the

From the Department of Internal Medicine, National Defense Medical College, Tokorozawa, Japan.

Address for correspondence: Akira Kurita, MD, Department of Internal Medicine, National Defense Medical College, 3-2 Namiki, Tokorozawa 359, Japan.

Received for publication March 18, 1996.

Accepted May 16, 1996. 
implantation of an autonomic defibrillator has disadvantages. ${ }^{7-11)}$ Inoue et al ${ }^{12)}$ reported the usefulness of ethyl alcohol or phenol directly injected into coronary artery in ablating VT. Although several investigators ${ }^{13,14)}$ modifying their method using ethyl alcohol reported that it terminates VT, clinical application of this procedure is difficult because the technique of infusing ethanol into a coronary artery can lead to necrosis in normal and unrelated regions of the endocar$\operatorname{dium}^{15)}$

Direct subendocardial injection of ethyl alcohol via a catheter was first reported by Weismuller et $\mathrm{al}_{,}{ }^{16)}$ who found that their procedure led to a controlled local lesion. However, they did not examine the procedure with respect to its possible usefulness in controlling VT. Therefore, the present study was designed to determine whether or not the direct injection of ethyl alcohol into the myocardium, wherein a lesion controlled for location and size would be inflicted, could be used to terminate VT without causing unnecessary damage in other areas of the myocardium. To accomplish this purpose, we used an endoscopic needle catheter to inject ethanol into the myocardium of a VT model following a technique that is used successfully in sclerotherapy for esophageal varices. ${ }^{17,18)}$

\section{Materials and Methods}

Epicardial experiment: Seventeen mongrel adult dogs of either sex, weighing 13-28 kg, were anesthetized with ketamine hydrochloride, $2 \mathrm{mg} / \mathrm{kg} \mathrm{IM}$ followed by pentobarbital $(30 \mathrm{mg} / \mathrm{kg}, \mathrm{IV})$; additional doses were administered as needed to maintain deep anesthesia. The dogs were intubated with a cuffed endotracheal tube and ventilated with room air using a Harvard respirator. Under positive pressure ventilation, the chest was opened through a left thoracotomy at the fourth or fifth intercostal space and the heart was suspended in a pericardial cradle. A fluid-filled cannula was inserted into the left cervical artery and connected to a transducer (Statham $\mathrm{p}-23 \mathrm{~dB}$ ) to monitor arterial pressure, and a left-cervical venous cannula was used to infuse normal saline at $30 \mathrm{~m} l / \mathrm{h}$ to replace spontaneous fluid loss. A $1 \mathrm{~m} l$ syringe with a 26 -gauge needle was carefully placed in the cpicardium of the area between the first and second diagonal branch of the left anterior descending (LAD) coronary artery (Figure 1) in preparation for aconitine administration. Surface electrocardiographic (ECG) lead II and arterial pressure were recorded throughout the experiment. A left ventricular electrogram (LVE) was also recorded using a bipolar electrogram catheter inserted from the right femoral artery. Recordings were made on a Nihonkoden recorder (RMC 1100) at paper speeds of 5, 10, 25, 50 and $100 \mathrm{~mm} / \mathrm{sec}$, as needed. Recordings from Marquette Holter ambulatory monitoring leads, aVf and CM5, were maintained throughout the experiment. Once the animals had 


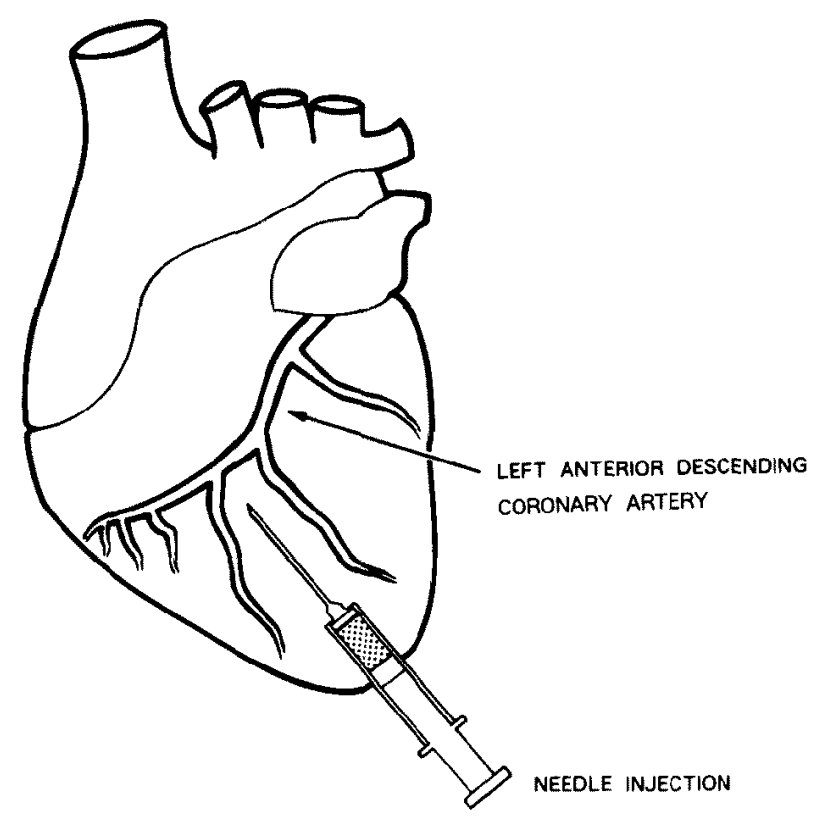

Figure 1. Site of aconitine administration used to induce VT during open heart surgery in the dog, and of ethanol administration using direct injection into the epicardium to suppress the induced VT.

been fully connected to the instruments, and after a control recording time period of at least $10 \mathrm{~min}, 1.0 \mu \mathrm{g}$ (median dose; range 0.25 to $3.75 \mu \mathrm{g}$ ) of aconitine was carefully injected into the epicardium until VT was provoked. After the VT had persisted for at least 3 minutes, $0.6 \mathrm{~m} l$ (median dose; range $0.2-2.0 \mathrm{~m} l$ ) of $96 \%$ ethanol was carefully injected into the same epicardial region through a separate 26-gauge needle until the normal sinus rhythm reappeared. Recovery from VT to normal sinus rhythm was determined by the appearance of a normal rhythm that lasted for at least $3 \mathrm{~min}$ while the animal was hemodynamically stable, as defined previously. ${ }^{19)}$ The return to normal sinus rhythm and hemodynamic stability was confirmed by the failure of normal saline injected through the needle to alter these physiological events. Recording from the Holter ambulatory monitoring leads also confirmed the recovery from VT. If the systolic blood pressure fell below $90 \mathrm{mmHg}, 2$ to $3 \mathrm{ml}$ of methoxamine $(0.1$ to $0.3 \mathrm{mg} / \mathrm{kg})$ were administered IV. The alpha adrenergic receptor agonist effects of methoxamine were expected to increase blood pressure and maintain the coronary perfusion pressure by prolonging the diastolic phase and slowing the heart rate. The dogs were sacrificed with a lethal IV dose of pentobarbital at the end of the experiment and while still under deep anesthesia. The hearts were examined for acute morphologic damage and samples of left ventricular tissue were obtained from the morphologically damaged areas and from the visibly normal surrounding 
tissue. Gross measurements were made (in $\mathrm{mm}$ ) of the greatest length, width, and depth of the acute lesions; the necrotic areas produced by ethanol were easily recognized macroscopically. Assuming that the area of the lesion is that of an ellipse and the volume of the lesion is that of a flattened rotated ellipsoid, the size of the lesion was calculated as follows: area $=$ length $\times$ width $\times \pi / 4$; volume $=$ area $\times$ depth $/ 1.5$. Thereafter, tissues were removed and fixed in formalin for at least two weeks before being examined histologically. Lesioned areas were sectioned and stained with phosphotungstic acid hematoxylin-eosin and Masson's trichrome stain to assess myocardial injury.

Endocardial experiment: Thirteen mongrel dogs were anesthetized as described above. After each dog was intubated and ventilated, a fluid-filled cannula was inserted into the femoral artery to monitor arterial pressure. A bipolar catheter to record the left ventricular electrogram was placed in the left ventricle from the right femoral artery, as described above. A 23-gauge needle catheter (Variocath type $\mathrm{C}$ ) that is used to cauterize esophageal varices was then carefully introduced into the left ventricle using a 9F guiding catheter. As shown in Figure 2 , the tip length of the needle could be controlled (from 1 to $5 \mathrm{~mm}$ ) using a nut adjustment. Aconitine mixed with $0.2 \mathrm{ml}$ contrast medium, in an amount equivalent to that used in the epicardial experiment, was carefully injected into the endocardium through the needle catheter. This was carried out under fluoroscopic control after pushing the needle 1-2 mm out of the lumen of the guiding catheter (Figure 3). The criteria for VT were the same as in the epicardial injection experiment. After VT was induced, the needle catheter was quickly withdrawn and changed to another 23-gauge needle catheter, to be used for the ethanol injection. The needle catheter was carefully introduced into the left ventricle using the same $9 \mathrm{~F}$ guiding catheter and the needle tip was extended out to the same length as before. The tip could not penetrate the myocardium since the length of the needle tip was controlled by a nut adjustment. The positions of the
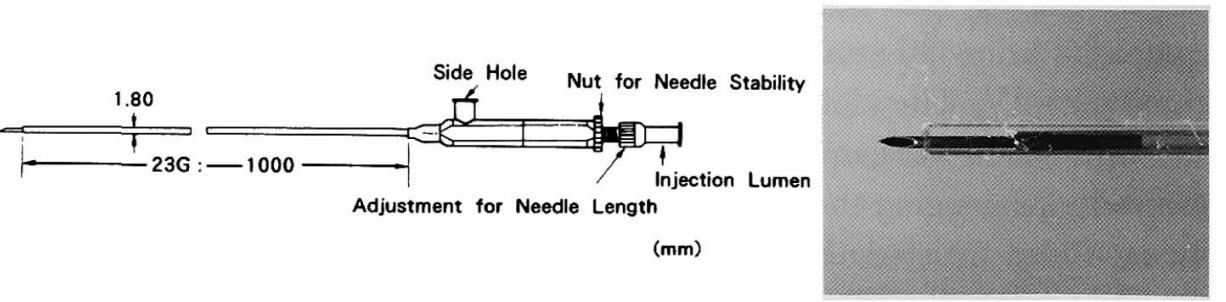

Figure 2. Variocath type $\mathrm{C}$ needle catheter. Tube length was $1000 \mathrm{~mm}$ and lumen diameter was $1.80 \mathrm{~mm}$ (left); tip of needle showing $2 \mathrm{~mm}$ out of guiding catheter (right). 


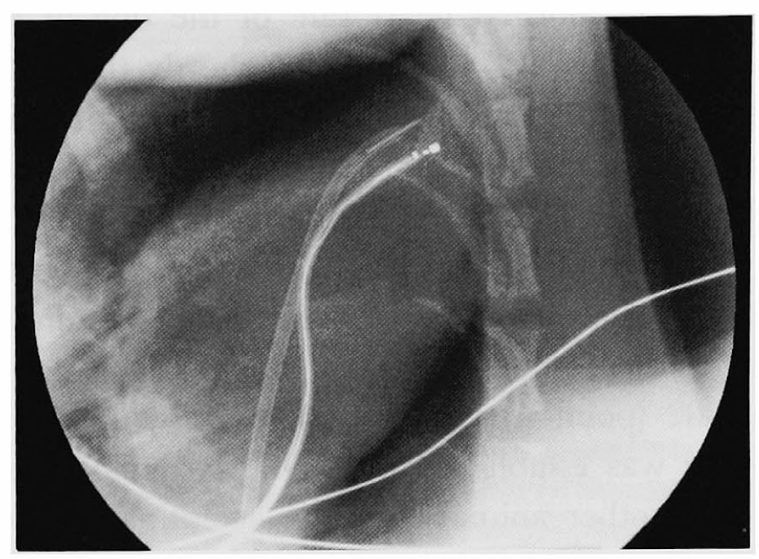

Figure 3. The position of tip of needle from the Variocath and the position of the bipolar electrogram catheter in the left ventricle.

needle tips were the same during all procedures. After the duration of VT was confirmed, the same amount of $96 \%$ cthanol was injected into the endocardium until the VT was replaced by a regular sinus rhythm. Both EGG and systemic hemodynamics were monitored during these manipulations. Procedures for euthanization, inspection of morphologic damage, and preparation of tissue for histology necrosis were the same as those described in the epicardial experiments Analysis of data: Data are expressed as mean $\pm \mathrm{SD}$ or median and range. Differences between means were analyzed by paired and unpaired t-tests and a level of $p<0.05$ was considered statistically significant. The doses of ethanol and duration of VT were analyzed with linear regression analysis.

\section{Results}

Epicardial study; Induction and termination of VT: Administration of 1.0 $\mu \mathrm{g}$ (median dose) of aconitine induced VT in the 17 dogs (Table I). After VT had persisted for at least $3 \mathrm{~min}, 0.6 \mathrm{ml}$ (median dose) of $96 \%$ ethanol at $21^{\circ} \mathrm{C}$ administered to the epicardial region of each dog terminated VT in 15 of the dogs (group A); the remaining 2 (group B) died of ventricular fibrillation (VF), showing a consequent fall in both systolic and diastolic blood pressure (Table I). In the 15 dogs in Group A, a regular sinus rhythm was restored after the ethanol injection and stable hemodynamics persisted for at least $30 \mathrm{~min}$ thereafter. There were no differences between groups A and B with respect to cycle length (pre- and during VT) or blood pressure during VT. Data on each of the dogs used in the study are presented in Table I. The sequence of the induction and suppression of VT in a representative $\operatorname{dog}(\# 5)$ is shown in the Holter monitoring record (Figure 4). The 
first three lines show the normal heart rate of the dog at the outset of the procedure, starting at 15:35. VT was induced shortly thereafter 15:38 (arrow) and was fully apparent and steady by 15:40. The administration of ethanol at 15:46 (point 1) was followed by an immediate cessation of VT for the remainder of that minute. However, VT resumed during the next minute (15:47) and continued until a further dose of ethanol was administered at time 15:50 (point 2). VT reappeared shortly thereafter and ethanol was again administered at 15:56 (point 3). Following a brief respite, VT resumed and a fourth dose of ethanol was administered at 16:02 (point 4). Thereafter, VT gradually diminished and a regular sinus rhythm was established by 16:07; systemic hemodynamics were stable thereafter. In another animal (\#8), the aconitine induced VT was converted into a regular sinus rhythm immediately after ethanol administration into the epicardium. At the same time, systolic blood pressure rose dramatically from 74 to $100 \mathrm{mmHg}$ (Figure 5). Gross and histologic examinations revealed myocar-

Table I. Data for the 17 Dogs Used in the Epicardial Study

\begin{tabular}{|c|c|c|c|c|c|c|}
\hline \multirow{2}{*}{$\begin{array}{c}\text { Dog } \\
\text { number }\end{array}$} & \multirow{2}{*}{$\begin{array}{c}\text { Aconitine } \\
(\mu \mathrm{g})\end{array}$} & \multirow{2}{*}{$\begin{array}{l}\text { Ethanol } \\
(\mathrm{m} l)\end{array}$} & \multicolumn{3}{|c|}{ Cycle length (msec) } & \multirow{2}{*}{$\mathrm{BP}(\mathrm{VT})$} \\
\hline & & & Pre VT & During $\mathrm{VT}$ & Post VT & \\
\hline (1) & 1.0 & 0.6 & 460 & 224 & 510 & $149 / 98$ \\
\hline (2) & 1.75 & 1.6 & 450 & 240 & 410 & $118 / 78$ \\
\hline (3) & 0.75 & 2.0 & 380 & 208 & 400 & $142 / 112$ \\
\hline (4) & 0.25 & 1.0 & 352 & 108 & 304 & $159 / 101$ \\
\hline (5) & 1.75 & 0.8 & 330 & 184 & 350 & $107 / 87$ \\
\hline (6) & 1.0 & 0.6 & 450 & 262 & 460 & $105 / 64$ \\
\hline (7) & 2.75 & 0.2 & 400 & 272 & 328 & $126 / 85$ \\
\hline (8) & 1.0 & 0.6 & 450 & 320 & 492 & $74 / 59$ \\
\hline (9) & 0.75 & 0.4 & 500 & 175 & 507 & $126 / 100$ \\
\hline$(10)$ & 2.0 & 0.4 & 490 & 260 & 480 & $150 / 70$ \\
\hline (11) & 0.75 & 0.4 & 520 & 260 & 320 & $119 / 103$ \\
\hline (12) & 1.0 & 0.6 & 380 & 210 & 400 & $105 / 82$ \\
\hline (13) & 1.0 & 0.6 & 460 & 260 & 500 & $107 / 60$ \\
\hline (14) & 1.25 & 0.2 & 360 & 210 & 340 & $93 / 62$ \\
\hline \multirow[t]{2}{*}{ (15) } & 3.75 & 0.4 & 520 & 170 & 480 & $140 / 100$ \\
\hline & $1.51 \pm 1.02$ & $1.05 \pm 1.0$ & $433 \pm 62$ & $224 \pm 52$ & $419 \pm 77$ & $121 \pm 23 / 84 \pm 18^{*}$ \\
\hline \multicolumn{7}{|c|}{ (B) Group B (death by VF) } \\
\hline (1) & 1.25 & 0.4 & 304 & 216 & VF & $95 / 72$ \\
\hline \multirow[t]{2}{*}{ (2) } & 0.75 & 0.2 & 390 & 200 & VF & $51 / 28$ \\
\hline & $1.0 \pm 0.36$ & $0.3 \pm 0.15$ & $347 \pm 61$ & $208 \pm 11$ & & $73 \pm 31 / 50 \pm 31$ \\
\hline \multicolumn{7}{|c|}{$\begin{array}{l}\text { Data include amounts of aconitine needed to induce VT, doses of } 96 \% \text { ethyl alcohol administered to the } \\
\text { epicardium to suppress the induced VT, cycle lengths and blood pressures. Suppression of VT was successful in } \\
15 \text { dogs (A); the other } 2 \text { (B) died following ventricular fibrillation. }\end{array}$} \\
\hline
\end{tabular}




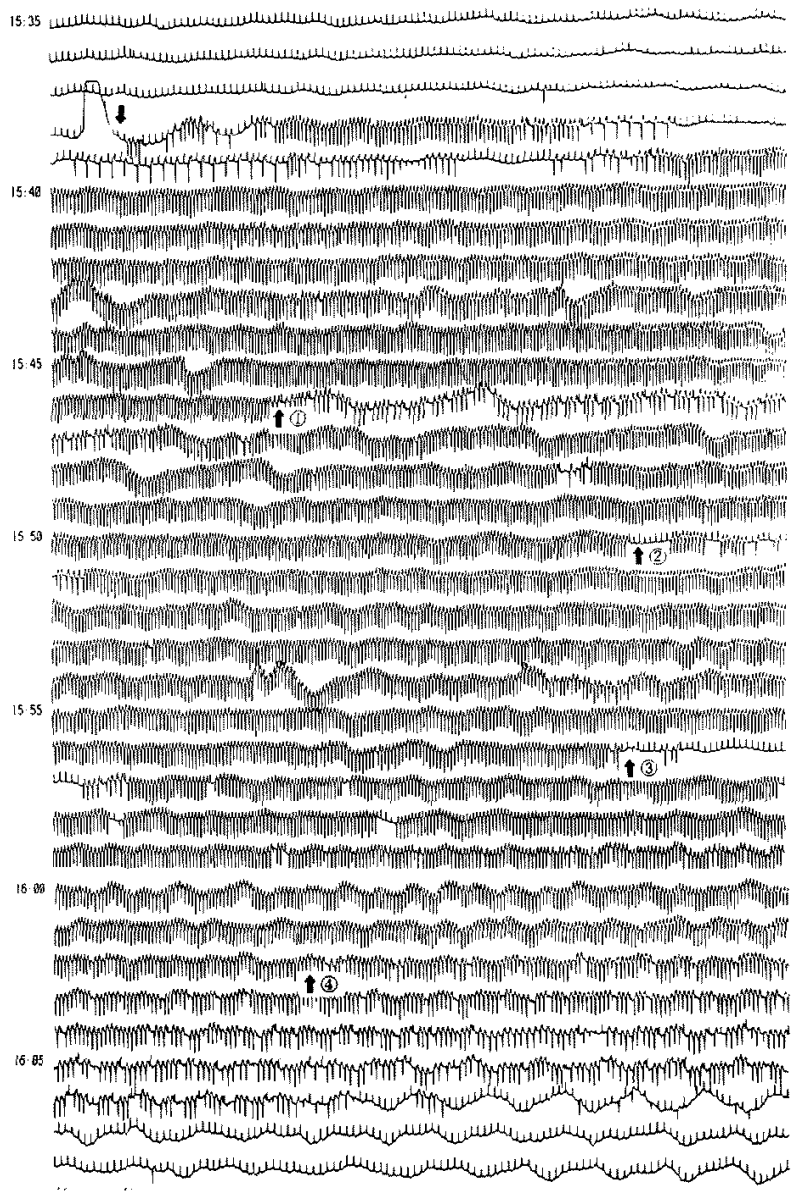

Figure 4. The Holter monitoring record of a representative case (dog $\# 5$ ) from time 15:35 through 16:08. VT was induced by aconitine injection during 15:53 (arrow) and disappeared gradually after administration of $96 \%$ ethanol into the epicardium (15:46, point 1). After several injections of $96 \%$ ethanol into the epicardium (points 2, 3 and 4 ), the VT was completely gone by $16: 07$.

dial necrosis following the administration of ethanol in all dogs studied. The necrosis was observed in all animals, i.e., those that reverted to regular sinus rhythms (group A, $n=15$ ) and those that died of VF (group B, $n=2$ ) following the epicardial administration of ethanol. Histologic examination showed no transmural necrosis and no luminal fibrin or thromboses in either group. The volume of necrosis in the dogs that reverted to regular sinus rhythm appeared to be larger than in those that died of VF (Table II). Further, neither the depth nor the maximum diameters of the regions were different in the two groups (Table II). A typical example of the macroscopic extent of the lesion is shown in Figure 6 , and a typical example of the microscopic examination by $\mathrm{HE}$ staining is shown in Figure 7. 

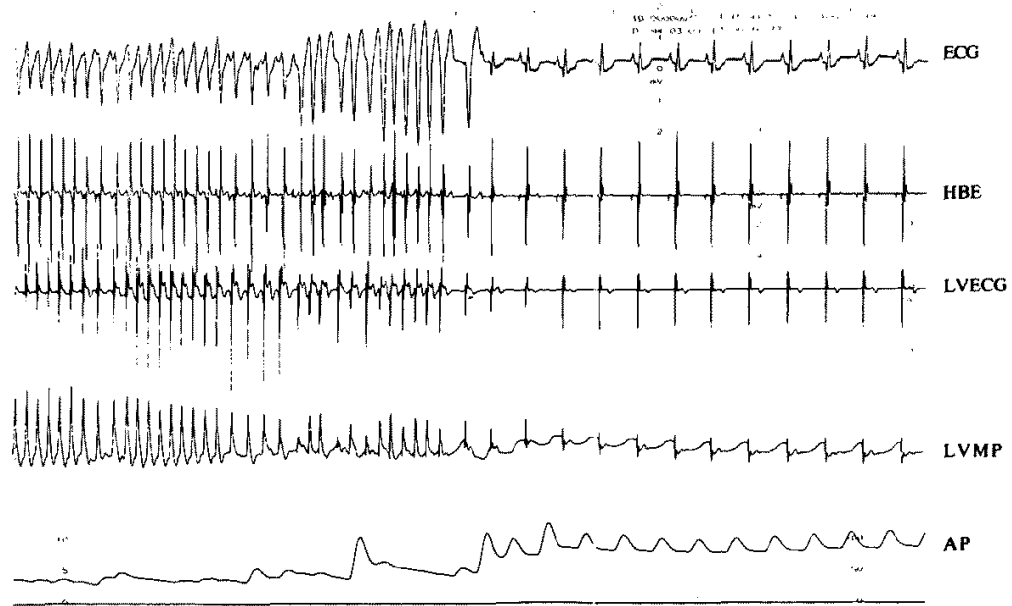

Figure 5. The record of a case (dog \#4) wherein the induced VT was converted to a normal sinus rhythm immediately after an ethyl alcohol injection into the epicardium. Systolic blood pressure immediately increased from around $50 \mathrm{mmHg}$ to 100 $\mathrm{mmHg} . \mathrm{ECG}=$ surface electrocardiography; $\mathrm{HBE}=\mathrm{His}$ bundle $\mathrm{ECG} ; \mathrm{LVECG}=\mathrm{left}$ ventricular ECG; LVMP = LV monophasic potential ECG; AP = aortic pressure.

Table II. Characteristics of the Lesions in the Epicardial Study

\begin{tabular}{lcc}
\hline & $\begin{array}{c}\text { Group A dogs } \\
(n=8)\end{array}$ & $\begin{array}{c}\text { Group B dogs } \\
(n=2)\end{array}$ \\
\hline Volume $\left(\mathrm{mm}^{3}\right)$ & $61 \pm 47$ & $124 \pm 92.0$ \\
Depth $(\mathrm{mm})$ & $1.5 \pm 0.5$ & $1.3 \pm 1.0$ \\
Maximum diameter $(\mathrm{mm})$ & $15.6 \pm 8.5$ & $14.5 \pm 10.8$ \\
\hline
\end{tabular}

Group A (converted to regular sinus rhythm), Group B (death by VF), Values are mean \pm SD.

Endocardial study; Induction and termination of VT: The administration of aconitine, $1.75 \mu \mathrm{g}$ (median dose; range $1.0-23.75 \mu \mathrm{g}$ ) induced VT in all $13 \mathrm{dogs}$ (Table III); this dosage of aconitine was not significantly different from that used in the epicardial experiments. VT in 7 of the 13 dogs was converted to a regular sinus rhythm after administration of $2.0 \mathrm{ml}$ of ethanol (median; range, 0.6-3.5 $\mathrm{ml}$ ), a dose that was not significantly different from that used for the epicardial studies. The remaining 6 dogs failed to respond to ethanol administration and died of VF. Cycle lengths in the two groups before and during VT were not significantly different, but systolic and diastolic blood pressures in group C were significantly higher than in group D during VT (Table III). Gross and histologic examinations revealed a necrosis similar to that created by epicardial injection of ethanol. Neither the depth nor the maximum diameters of the lesions were different in the two groups. The size of necrotic areas of those that died of VF were not significantly different from those that recovered to a regular sinus rhythm (Table IV). In both the epicardium and endocardium studies, doses of ethanol in dogs 


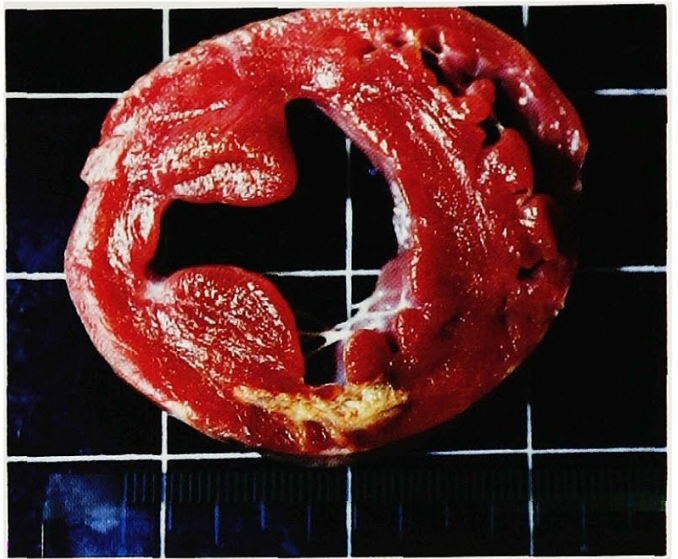

Figure 6. Transverse ventricular slices showing the typical endocardial necrosis (whitish yellow) induced by the administration of $96 \%$ ethanol; dog \#5 (group C) of endocardial study.

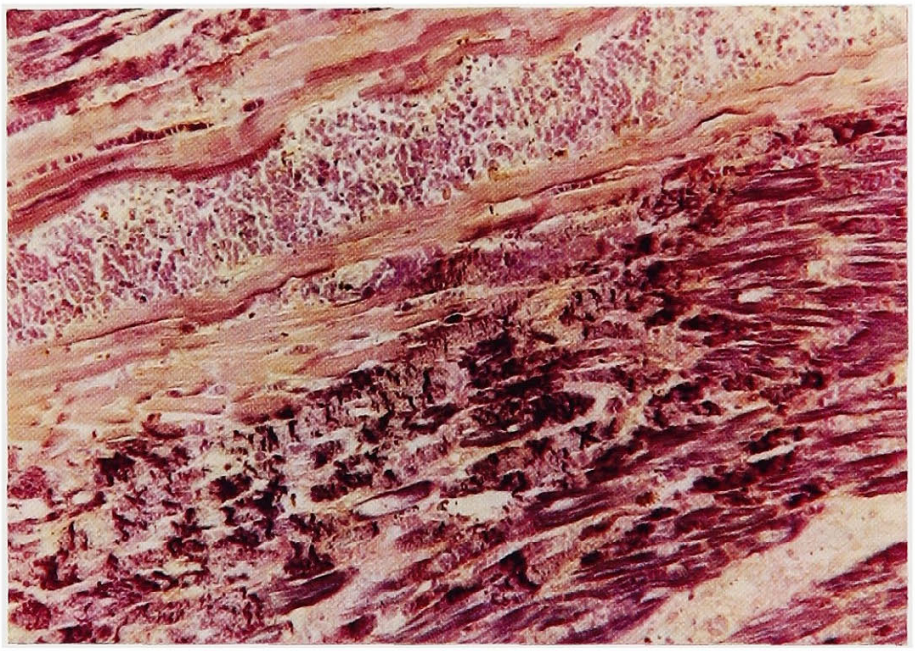

Figure 7. Histologic examination of the left ventricle of dog \#5 showing necrosis of myocardial cells with disarray and dislodgment. (Phosphotungstic Acid Hematoxylin Eosin staining)

that recovered to normal sinus rhythm were compared to the ethanol doses of the dogs that died of VF; there was no significant difference between the groups (Table V). Further, there was no significant relationship between the dose of ethanol and VT duration (Figure 8). 
Table III. Data for the 13 Dogs Used in the Endocardial Study

\begin{tabular}{|c|c|c|c|c|c|c|}
\hline \multirow{3}{*}{$\begin{array}{c}\text { Dog } \\
\text { number }\end{array}$} & \multirow{3}{*}{$\begin{array}{l}\text { Aconitinte } \\
\qquad(\mu \mathrm{g})\end{array}$} & \multirow{3}{*}{$\begin{array}{l}\text { Ethanol } \\
(\mathrm{m} l)\end{array}$} & \multirow{2}{*}{\multicolumn{3}{|c|}{ Cycle length (msec) }} & \multirow{3}{*}{$\mathrm{BP}(\mathrm{VT})$} \\
\hline & & & & & & \\
\hline & & & Pre VT & During VT & Post VT & \\
\hline (1) & 1.0 & 0.6 & 430 & 260 & 420 & $149 / 98$ \\
\hline (2) & 23.75 & 0.6 & 460 & 290 & 390 & $118 / 78$ \\
\hline (3) & 15 & 3.0 & 530 & 230 & 610 & $125 / 80$ \\
\hline (4) & 15 & 1.6 & 418 & 270 & 440 & $69 / 52$ \\
\hline (5) & 0.5 & 2.0 & 404 & 141 & 500 & $76 / 69$ \\
\hline (6) & 1.0 & 2.0 & 468 & 185 & 544 & $137 / 57$ \\
\hline \multirow[t]{2}{*}{ (7) } & 2.0 & 3.5 & 383 & 157 & 410 & $144 / 99$ \\
\hline & $8.3 \pm 9.5$ & $1.90 \pm 1.10$ & $442 \pm 49$ & $219 \pm 59$ & $473 \pm 81$ & $116 \pm 32^{*} / 76 \pm 10^{*}$ \\
\hline \multicolumn{7}{|c|}{ (D) Group D (death by VF) } \\
\hline (1) & 1.75 & 0.5 & 520 & 230 & $\mathrm{VF}$ & $68 / 50$ \\
\hline (2) & 12.5 & 2.1 & 360 & 150 & $\mathrm{VF}$ & $56 / 40$ \\
\hline (3) & 1.25 & 5.0 & 380 & 260 & $\mathrm{VF}$ & $84 / 60$ \\
\hline (4) & 0.5 & 1.0 & 413 & 180 & $\mathrm{VF}$ & $109 / 82$ \\
\hline (5) & 1.25 & 5.0 & 430 & 83 & VF & $31 / 6$ \\
\hline \multirow[t]{2}{*}{ (6) } & 8.75 & 3.0 & 390 & 76 & VF & $46 / 9$ \\
\hline & $4.33 \pm 5$ & $2.77 \pm 1.94$ & $416 \pm 57$ & $163 \pm 75$ & & $66 \pm 30 / 42 \pm 30$ \\
\hline
\end{tabular}

Data include amounts of aconitine needed to induce VT, doses of $96 \%$ ethyl alcohol administered to the endocardium to suppress the induced VT, cycle lengths and blood pressures. Suppression of VT was successful in 7 dogs $(\mathrm{C})$; the other $6(\mathrm{D})$ died following ventricular fibrillation.

$\mathrm{VT}=$ ventricular tachycardia; $\mathrm{VF}=$ ventricular fibrillation; $\mathrm{BP}=$ blood pressure $(\mathrm{mmHg})$ during $\mathrm{VT}$. ${ }^{*} p<0.05$ (group C vs. group D), Values are mean \pm SD.

Table IV. Characteristics of the Lesions in the Endocardial Study.

\begin{tabular}{lccc}
\hline & $\begin{array}{c}\text { Group C } \\
(n=4)\end{array}$ & $\begin{array}{c}\text { Group D } \\
(n=4)\end{array}$ & $p$ values \\
\hline Volume (mm3) & $69 \pm 40$ & $126 \pm 92$ & $\mathrm{~ns}$ \\
Depth (mm) & $2.5 \pm 0.5$ & $1.3 \pm 0.5$ & $\mathrm{~ns}$ \\
Maximum diameter $(\mathrm{mm})$ & $7.6 \pm 3$ & $8 \pm 5$ & $\mathrm{~ns}$ \\
\hline
\end{tabular}

Group C (converted to regular sinus rhythm), Group D (death by VF), Values are mean \pm SD

Table V. Epicardial and Endocardial Studies: Ethanol Doses and VT Duration in Dogs that Survived (wherein VT was Converted to Regular Sinus Rhythm) and Dogs that Died of VF

\begin{tabular}{lccc}
\hline & $\begin{array}{c}\text { Converted to regular sinus } \\
\text { rhythm } \\
(n=22)\end{array}$ & $\begin{array}{c}\text { Death by VF } \\
(n=8)\end{array}$ & $p$ values \\
\hline Ethanol $(\mathrm{ml})$ & $1.76 \pm 2.3$ & $2.2 \pm 1.9$ & $\mathrm{~ns}$ \\
VT duration (scconds) & $154 \pm 156$ & $79 \pm 95$ & $\mathrm{~ns}$ \\
\hline
\end{tabular}

Values are mean $\pm \mathrm{SD}$. 


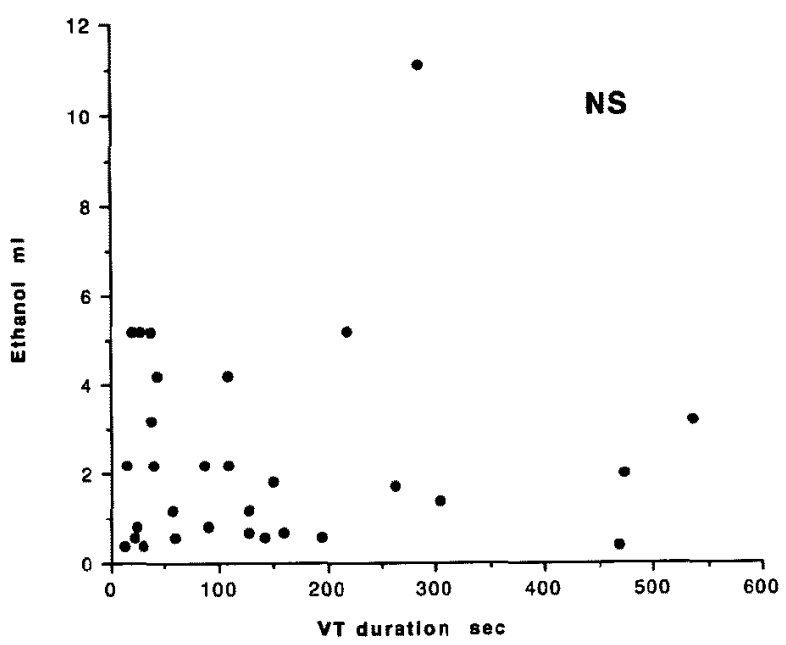

Figure 8. Correlation of ethanol doses $(\mathrm{m} l)$ versus VT duration (seconds).

\section{Discussion}

In this study, 15 dogs recovered from aconitine-induced VT after ethanol was injected directly into the epicardium, while 2 others died of VF following aconitine administration. In addition, 7 of the 13 dogs recovered from aconitineinduced VT after ethanol was injected into the endocardium with the Variocath type-C needle catheter, while 6 others died of VF. Pathologic examination suggested that injection of $96 \%$ ethanol directly into the epi- and endocardium created a necrotic myocardial region that could terminate the aconitine-induced VT. By keeping the size of the lesion small, effective VT suppression could be accomplished without inducing severe hypotension. In the surviving dogs, the blood pressures in both epi- and endocardial experiments were significantly higher than those pressures recorded in dogs that died of VF during the experiment. Therefore, the VF that led to the deaths probably resulted from a secondary subendocardial ischemia caused by hypotension. This seems likely because the blood pressure in these dogs could not be maintained despite the infusion of methoxamine.

Recent basic and clinical studies indicate that lesions induced by intracoronary injection of $50-100 \%$ ethanol can terminate VT. Inoue et al ${ }^{12)}$ first reported that intra-coronary injection of $25 \%$ phenol or $50-100 \%$ ethanol could suppress aconitine-induced VT in dogs. Gursoy et al ${ }^{20)}$ described the clinical use of ethanol injections into coronary arteries in treating recurrent sustained VT, and reported that ethanol injected into a tachycardia-related artery successfully terminated VT. However, a transient elevation of creatine phosphokinase (CPK) and chest pains followed shortly thereafter. De Paola et $\mathrm{a}^{21)}$ also reported on the 
use of $96 \%$ ethanol to generate transcoronary chemical ablations to control VT in chronic Chagas myocarditis; they claimed to successfully induce an ablation in the tachycardia-related coronary artery without a consequent acute clinical or laboratory deterioration of left ventricular function. However, because the proximal coronary artery supplies a large area, its use for ethanol infusion risks damage to an excessive amount of myocardium. Instead, intracoronary infusion of ethyl alcohol should be used in only selected instances, such as those in which there is an anatomically well-defined area of arrhythmogenic VT and where the infused coronary artery supplics the peripheral artery. In such cases there would be no chance of massive myocardial necrosis that could elevate the ST segment or the CPK. Surgical interventions for the treatment of VT have involved cryoablation techniques and direct transmural ventriculostomy. Cryosurgery for ablation of sustained monomorphic VT due to coronary artery disease has proven to be a useful procedure. However, since the depth of a cryolesion depends upon the duration and degree of freezing, the procedure can increase the duration of an operation. In any event, the depth of the lesion is difficult to evaluate with current techniques. ${ }^{22)}$ Direct transmural ventriculostomy is a useful procedure for recurrent VT, but it poses a higher mortality risk than does ICD implantation $^{237}$ and it requires an open heart endocardial resection approach that involves peeling off a layer of endocardium and/or often the resection of the rim of an aneurysm. ICD devices are now markedly improved over what they were in the past. Kron et $\mathrm{al}^{24)}$ and others ${ }^{8-11\}}$ reported that ICD can be used for treating VT, and a randomized study of the use of an ICD for controlling VT in patients 2-4 weeks after MI has shown its effectiveness. ${ }^{25)}$ However, such equipment is in the trial stage, and it is still to be determined whether or not an ICD is better than antiarrhythmic agents. ${ }^{26)}$ One disadvantage is that the generator must be changed every few years, depending on its frequency of usage. Furthermore, an ICD is costly and somewhat risky. Nunain et $\mathrm{al}^{27)}$ reported that hardware-related complications and/or inappropriate shocks have occurred in patients after ICD devices were implanted. Therefore, patients implanted with ICD devices may be subjected to additional and unnecessary physical stress. Weismuller et al ${ }^{15}$ reported on the feasibility of chemical ablation by a subendocardial injection of ethanol via a catheter. They recognized the possibility of generating a local necrotic area of limited and controlled size after 25 days of initial chemical ablation, and they further suggested the possibility of chemical ablation by direct injection into the endocardium. They also reported that the necrotic region created by an ethanol injection into the endocardium does not impair left ventricular function after 3 weeks because the initial zone of necrosis is nearly completely subsumed by retracting connective tissue. The present data suggest that both direct epi- and endocardial injection of ethanol can terminate VT, possibly by 
inducing ablation in the VT related epi- and endocardial region. However the doses of ethanol in those cases where VT was replaced by regular sinus rhythms were not significantly greater than in those cases where dogs died of VF. Further, there was no significant relationship between the dose of ethanol and VT duration. Thercfore, optimal cthanol doses could not be determined in this study. The technique of ethanol administration uscd in the present study may be a more suitable procedure than radiofrequency ablation for a VT that originates at a deep region of the endocardium, since radiofrequency ablation cannot reach intermediate or epicardial regions. The present method may be superior for use in polymorphic VT because the necrotic region is rather large, although after several weeks necrotic regions might be replaced by retracting connective tissue.

There are several limitations in the present study. First, aconitine-induced VT differs from VT observed in patients. Aconitine-induced VT is believed to stem from enhancement after depolarization due to cathodal currents or in response to low external $\mathrm{Ca}^{++}$concentrations, which in turn cause altered $\mathrm{Na}^{+}$and $\mathrm{Ca}^{++}$exchange ${ }^{28)}$ Therefore, the model used in the present study does not necessarily apply to other cases of VT that stem from depolarization induced automatically in the Purkinje fibers or ventricular muscle. Further, studies of chronic infarction with inducible VT in an animal model are also required to demonstrate the applicability and effectiveness of transluminal chemical catheter ablation in this situation. This study was limited in scope to a model in which recovery from VT was not followed beyond several hours.

Second, the injection of ethanol directly into the ventricular epi- and endocardium does not always result in ethanol entering the myocardium exclusively; some of the fluid may leak into the surrounding areas. This is substantiated by our finding that the amounts of ethanol injected via the endocardial approach may have been slightly greater than the amounts injected via the epicardial approach. However, there were no serious hemodynamic effects. Nevertheless, this point should be examined in detail in subsequent studies. Third, the possibility of perforation should be considered. In the Weismuller et al $^{15)}$ study, perforation occurred twice but there were no further complications. We did not encounter any perforations in the present study, possibly because we employed a fine needle catheter that is used clinically in our hospital for the treatment of esophageal varix and gastric ulcer. Nevertheless, in a clinical setting, application of this technique in cases having a thin ventricle (e.g., dilated cardiomyopathy) should be avoided because of the increased risk of perforation; further adjustment to make an appropriately sized lesion might be difficult.

Despite these limitations, the present pilot study suggests that such a technique might be feasible in a clinical setting. This would be true if a catheter is specially designed so that the balloon in the catheter tip is kept in the same 
position inside the ventricle to minimize leakage of ethanol. In addition, this technique could be useful if conventional methods, such as antiarrhythmic agents and/or radio-frequency techniques, are unsuccessful.

In summary, VT induced by direct myocardial injection of aconitine into anesthetized dogs can be converted to regular sinus rhythm by direct epicardial or endocardial injection of ethanol into the same myocardial region. These preliminary data suggest the utility of this chemical ablation technique for terminating VT.

\section{REFERENCES}

1. Swerdow CD, Winkle RA, Mason JW. Determinations of survival in patients with ventricular tachyarrhythmias. N Engl J Med 1983; 308: 1436-42.

2. Wilber $\mathrm{JW}$, Garan $\mathrm{H}$, Finkelstein P. Out-of-hospital cardiac arrest: use of electrophysiological testing in the prediction of long-term outcome. N Engl J Med 1988; 318: 19-24.

3. Okumura K, Olshansky B, Henthiorn RW, et al. Demonstration of the presence of slow conduction during sustained ventricular tachycardia in man: Use of transient entrainment of the tachycardia. Circulation 1987; 75: 369-78.

4. Morady F, Frank R, Kou WH. Identification and catheter ablation of a zone of slow conduction in the reentrant circuit of ventricular tachycardia in humans. J Am Coll Cardiol 1988; 11: 775-82.

5. Ruder MA, Mead RH, Gaudiani V, et al. Transvenous catheter ablation of extranodal accessory pathways. J Am Coll Cardiol 1988; 11: 1245-53.

6. Morady F, Scheinman MM, Kou WH, et al. Long-term results of catheter ablation a posterior accessory atrioventricular connection in 48 patients. Circulation 1989; 79: 1160-70.

7. Harken AH, Josephson ME. Surgical management of ventricular tachycardia. In: Josephson ME, Wellens HJ, editors. Tachycardias: Mechanism, Diagnosis, Treatment. Philadelphia: Lea \& Febiger, 1984: 156 .

8. Tehou PJ, Kadri N, Anderson J, et al. Automatic implantable cardioverter defibrillators and survival of patients with left ventricular dysfunction and malignant ventricular arrhythmias. Ann Intern Med 1988; 109: 529-34.

9. Haines DE, Lerman BB, Kron IL, DiMarco JP. Surgical ablation of ventricular tachycardia with sequential map-guided subendocardial resection: electrophysiological assessment and long-term followup. Circulation 1988; 77: 131-41.

10. Winkle RA, Mead RH, Ruder MA, et al. Long-term outcome with the automatic implantable cardioverter-defibrillator. J Am Coll Cardiol 1989; 13: 1353-61.

11. Rosenthal ME, Josephson ME. Current status of antitachycardia devices. Circulation 1990; 82: 188999.

12. Inoue $\mathrm{H}$, Waller BF, Zipes DP. Intracoronary ethyl alcohol or phenol injection ablates aconitineinduced ventricular tachycardia in dogs. J Am Coll Cardiol 1987; 10: 1342-49.

13. Brugada $\mathrm{P}$, de Swart $\mathrm{H}$, Smeet $\mathbf{J}$, Wellens $\mathrm{HJJ}$. Transcoronary termination and ablation of ventricular tachycardia. Circulation 1989; 79: 475-482.

14. Nora MO, Miles WM, Klein LS, Dillon JC, Zipes DP. Alcohol ablation of ventricular tachycardia. J Cardiovasc Electrophysiol 1991; 2: 455-61.

15. Okishige K, Andrews TC, Friedman PL. Suppression of incessant polymorphic ventricular tachycardia by selective intracoronary ethanol infusion. PACE 1991; 14: 188-95.

16. Weismuller P, Mayer U, Richter P, Heieck F, Kochs M, Hombach V. Chemical ablation by subendocardial injection of ethanol via catheter-preliminary results in the pig heart. Eur Heart J 1991; 12: $1234-9$.

17. Kitano S, Kobayashi N, Iso Y, et al. Prevention of recurrence of csophageal varices after cndoscopic 
injection sclerotherapy with ethanolamine oleate. Hepatology $1987 ; 7: 810-5$.

18. Suzuki H, Masuda K, Ohmasa R, Akiba H, et al. Recent trends of endoscopic injection sclerotherapy for esophago-gastric varices. Gastrointest Endoscope 1990; 10: 1237-44 (in Japanese).

19. Kato R, Kurita A, Mitani H, Takase B, Nagayoshi H. Feasibility of a special designed needle catheter for the treatment of aconitine induced ventricular tachycardia in canine heart. 8th International Congress of Electrocardiography, 1994: 212 (abst).

20. Gursoy S, Nellens P, Guiraudon G, Brugada J, Brugada P. Epicardial and subselective transcoronary chemical ablation of incessant ventricular tachycardia. Cathet Cardiovasc Diagn 1993; 28: 323-7.

21. De Paola AA, Gomes A, Miyamoto MH, Martinez FO. Transcoronary chemical ablation of ventricular tachycardia in chronic Chagas myocarditis. J Am Coll Cardiol 1992; 20: 480-2.

22. Caceres J, Masood A, Werner $\mathrm{P}$, et al. Cryoablation of refractory sustained ventricular tachycardia due to coronary artery disease. Am J Cardiol 1989; 63; 296-300.

23. Lee R, Mitchell JD, Garan H, et al. Operation for recurrent ventricular tachycardia: Predictors of shortand long-term efficacy. J Thorac Cardiovasc Surg 1994; 107; 732-42.

24. KronJ, Silka MJ, Ohm OJ, Bardy G, Benditt D. Preliminary experience with nonthoracotomy implantable cardioverter defibrillators on young patients. PACE $1994 ; 17 ; 26-30$.

25. Wever EFD, Hauer RNW, van Capelle FJL, et al. Randomized study of implantable defibrillator as first choice therapy verus conventional strategy in post infarct sudden death survivors. Circulation 1995; 91 : 2195-203.

26. Zipes DP. Are implantable cardioverter-defibrillators better than conventional antiarrhythmic drugs for survivors of cardiac arrest? Circulation 1995; 91:2115-7.

27. Nunain SO, Roelke M, Osswald S, et al. Limitations and late complications of third-generation automatic cardioverter-defibrillators. Circulation 1995; 91: 2104-213.

28. Matsuda K, Hoshi T, Kameyama S. Effects of aconitine on the cardiac membrane potential of the dog. Jpn J Physiol 1959; 9: 419-29. 